
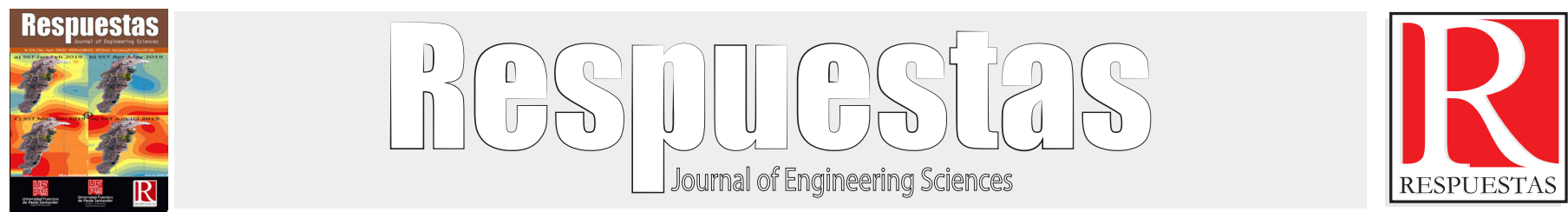

Original Article

https://doi.org/10.22463/0122820X.2954

\title{
Impacto laboral del graduado del programa de tecnología en procesos industriales de la Universidad Francisco de Paula Santander
}

\author{
Labor impact of graduate in technology in industrial processes of the Francisco de Paula Santander University \\ Pedro Garzón-Agudelo ${ }^{1}$, Yoneidy Benitez-García ${ }^{2}$, Wlamyr Palacios-Alvarado 3 \\ ${ }^{1}$ Especialista en gerencia de proyectos, ing.pedrogarzon@hotmail.com, orcid.org/0000-0003-0619-1701 Universidad Francisco de Paula Santander, Cúcuta - Colombia \\ ${ }^{2}$ Tecnólogo en Procesos Industriales, Ingeniero Industrial, benitezgarciayoneidy@gmail.com ., orcid.org/0000-0003-0619-1701 Universidad Francisco de Paula Santander, Cúcuta \\ - Colombia \\ ${ }^{3}$ Doctor en Ciencias Gerenciales, wlamyrpalacios@ufps.edu.co, http://orcid.org/0000-0002-4292-4178, Universidad Francisco de Paula Santander, Cúcuta-Colombia
}

Como citar: P. Garzón-Agudelo, Y. Benitez-García, W. Palacios-Alvarado "1Impacto laboral del graduado del programa de tecnología en procesos industriales de la Universidad Francisco de Paula Santander”. Respuestas, vol. 25, no. 2, pp. 125-132, 2020.

Received on February 07, 2020; Approved on April 5, 2020

\begin{tabular}{ll}
\hline & RESUMEN \\
\hline Palabras clave: & El presente proyecto se desarrolló con el propósito evaluar el impacto laboral del programa académico \\
& tecnología en procesos industriales de la Universidad Francisco de Paula Santander, tomando como base \\
el análisis de la percepción académica impartida en la formación profesional. Se aplicaron instrumentos de & recolección de información hacia las empresas del sector productivo de la región que mantienen vínculo con \\
Competencias, & el programa académico a través de diversas modalidades de extensión, permitiendo contrastar las necesidades \\
Fesempeño, & y expectativas demandadas por las organizaciones a nivel de competencias técnicas e integrales en su recurso \\
Graduados, & humano con las habilidades demostradas por los graduados del programa académico. Se determinó que la \\
Impacto laboral & inserción laboral en los graduados de programa se ve afectada por la preferencia que tienen éstos de continuar \\
& su formación en el ciclo profesional de Ingeniería. Así mismo se pudo establecer que gran parte de las \\
& Organizaciones del sector productivo de la región identifican a los graduados del programa de Tecnología en \\
& Procesos Industriales por su buen desempeño laboral medido desde la existencia de habilidades transversales. \\
& Finalmente se estableció un plan de acción que describe oportunidades de mejora en los procesos de docencia, \\
& a fin de mejorar continuamente la armonía entre las competencias de los nuevos graduados y las necesidades \\
de la sociedad en el ámbito regional.
\end{tabular}

\section{ABSTRACT}

\section{Keywords:}

Competences,

Formation,

Graduates,

Labour impact,

Performance,
The project was developed with the purpose of evaluating the labor impact of the academic program Technology in Industrial Processes of the Universidad Francisco de Paula Santander, taking as a base the analysis of the academic perception imparted in the professional training. Instruments were applied to collect information from companies in the productive sector of the region that maintain links with the academic program through various modalities of extension, allowing to contrast the needs and expectations demanded by organizations at the level of technical and comprehensive skills in their human resources with the skills demonstrated by graduates of the academic program. It was determined that the labor insertion in the graduates of the program is affected by their preference to continue their training in the professional cycle of Engineering. It was also possible to establish that a large part of the productive sector organizations in the region identify the graduates of the Industrial Process Technology program for their good work performance measured by the existence of transversal skills. Finally, an action plan was established that describes opportunities for improvement in the teaching processes, in order to continuously improve the harmony between the competencies of new graduates and the needs of society at the regional level.

*Corresponding author.

E-mail Address: ing.pedrogarzon@hotmail.com(Pedro Garzón-Agudelo) 


\section{Introducción}

El nivel educativo, le permite al ser humano desenvolverse con mayor facilidad en la sociedad y en los ámbitos laborales, debido a que genera las posibilidades para mejorar la calidad de vida y alcanzar metas y propósitos. Dadoque, porla educación es un procesogradual,mediante el cual se transmiten un conjunto de conocimientos, valores, comportamientos, actitudes y formas de actuar o pensar, que al ser adquiridos se emplearan en el trascurso de la existencia [1], para lograr desenvolverse de manera activa y participativa en la misma. Por lo cual cobra importancia la manera en que la formación se relaciona con el desarrollo económico. Así mismo la educación es entendida como un proceso de formación profesional y en el cual el aprendizaje permanente son pilares fundamentales para la consecución de empleo [2]; porque facilita las condiciones para aumentar la empleabilidad y contribuye con el desarrollo empresarial sostenible, donde la formación es la piedra angular del trabajo decente.

El Programa de Tecnología en Procesos Industriales de la universidad Francisco de Paula Santander, ha proyectado ofrecer las competencias cognitivas y comunicativas para formar talento humano capaz de gestionar y fortalecer procesos industriales, con el fin de garantizar que al culminar el proceso de formación, el graduado esté en la capacidad de aportar a que las empresas, alcancen un alto grado de productividad y competitividad, en un entorno ambientalmente sostenible, con la intención de incrementar la capacidad de innovación y desarrollo. Sin embargo, el éxito del proceso debe estar enmarcado en la calidad de la educación impartida en las instituciones desde el diseño curricular [3].

De allí que el Consejo Nacional de Acreditación (CNA), dentro de los criterios fundamentales requiere que se evalué el aspecto referente al seguimiento del graduado, cuyo valor y objetivo radica [4], en el contribuir a la explicación del desempeño profesional, posibilitando realizar un seguimiento continuo, que permita no sólo conocer la situación de los mismos en un momento determinado de tiempo, sino comparar su evolución, de acuerdo con el perfil académico y la situación del mercado laboral, evaluando el desempeño de los graduados y la percepción de la educación impartida por la institución, la aplicación de las competencias, [5] que permitan obtener una serie beneficios para la persona empleada y para la empresa donde se desempeña.

Bajo esta premisa, la finalidad de evaluar el impacto de la formación es que la información encontrada, contribuye con la toma de decisiones para ajustar las intervenciones, ampliarlas, recortarlas o sustituirlas [6]; siendo útil para los gestores de los programas y las entidades revisar, si el sistema de formación está orientado por la oferta, o es realmente una respuesta a la demanda, aspecto relevante para el programa en mención, que con el proyecto impacto laboral del graduado del Programa de Tecnología en Procesos Industriales, persigue datos valiosos para el fortalecimiento del mismo, a través del análisis de las fortalezas y las debilidades encontradas, contemplando los aspectos como, la situación laboral, el concepto que tiene el empleador y, el grado de pertenencia para con la institución, entre otros; en el ámbito académico, la opinión sobre la formación recibida, teniendo en cuenta al graduado como centro del proceso.

Investigaciones existentes hacen alusión a que, con el análisis de diversos estudios enfocados en el impacto de programas de académicos, el evaluar la pertinencia del programa académico se muestra como resultado la inserción en el mercado laboral y percepciones positivas del programa y la Institución [7]. La consolidación de la información se convierte en una oportunidad, para que el centro educativo enfoque su visión un poco más hacia empresarios, y no hacia la cultura del empleado actual [8]; y que el graduado ejerce un papel importante sobre el medio, con la creación de empresas, generación de empleos y desempeño óptimo en sus obligaciones laborales [9]. En este sentido, el artículo deja expuesto los resultados obtenidos tras la aplicación del estudio, destacando aquellos positivos, relacionados con el desarrollo de las competencias específicas, las cuales han sido definidas en el perfil profesional, el conocimiento del programa de Tecnología en Procesos Industriales en el mercado laboral y del desenvolvimiento de sus graduados, logrando percibir diferencias y similitudes por medio de comparativos entre la malla curricular del programa académico en cuestión, con el de otras universidades que contemplen o manejen el mismo programa o afines, considerando los requerimientos del sector productivo. Lo anterior permitirá, entre otras cosas, determinar cuáles asignaturas del plan de estudios son las que contribuyen en mayor medida al desarrollo de competencias laborales, y finalmente el planteamiento de alternativas que permitan la mejora continua de los 
procesos de formación impartidos.

\section{Materiales y Métodos}

El sistema educativo de la actualidad, cada día más exigente con las entidades de educación superior, destaca el reconocimiento del impacto laboral de los graduados en el medio, como un aspecto fundamental [10] para identificar el desempeño, la ubicación laboral, la disciplina y la coherencia de la formación recibida, en relación a las necesidades, el cual podrá utilizar tras la aplicación de múltiples estudios que persiguen el determinar si un programa produjo los efectos deseados en las personas e instituciones y si esos efectos se atribuyen a la intervención del programa [11].

Por lo anterior, el seguimiento del impacto surge como una herramienta tendiente a fortalecer la calidad de los programas, vista como un conjunto de características propias de la formación, donde se satisfacen las partes interesadas, a través de la capacidad de medir el impacto previsto, y aquel que no puede preverse [12]. Con la puesta marcha este tipo de proyectos, que podrán valerse de la utilización de una evaluación, cuyo diseño este enfocado en las unidades de observación, las unidades de análisis, los instrumentos de recolección de información, eligiendo los periodos de tiempo apropiados para llevarlos a cabo, después de una clara planificación [13], permitiendo validar el proceso formativo y un adecuado aporte al programa basado en la experiencia profesional [14]. Para lograr la validación del estudio, es vital entender, si la finalidad es caracterizar a los graduados del programa, o analizar el impacto que han tenido [15]

\section{Diseño Metodológico}

La investigación empleó descriptivo [16], además de combinar técnicas cualitativas y cuantitativas, para el tratamiento de datos, tras la aplicación de encuestas estructuradas a graduados y a empleadores de la región, lo que le agrega un enfoque mixto.

Para la aplicación de los instrumentos se consideraron la toma de dos clases de población, una enfocada en los graduados, con base es estadísticas del programa, correspondiente a 117 graduados de la Tecnología en Procesos Industriales, en el periodo comprendido entre 2010 y 2017. Se estimó un tamaño muestral para población finita, considerando la ecuación (1):

$$
\text { (1) } n=\frac{N * Z^{2} * p * q}{(N-1) * e^{2}+Z^{2} * p * q}
$$

\section{Donde}

n= Número de graduados del Programa de Tecnología en Procesos Industriales, correspondiente a 117 personas.

$Z=$ Nivel de confianza correspondiente del $95 \%$, con un $Z$ de 1,96 de confiablidad en los resultados

$p=$ Proporción de ocurrencia 0,5

$q=$ Proporción de rechazo 0,5

$e=$ Error o máxima diferencia entre la proporción muestral y la proporción de la población $=0,05$

Dando como resultado una totalidad de 90 graduados. La segunda población se refiere a las empresas donde han trabajado o llevado a cabo los proyectos de grado los graduados, bajo el registro soportado por el programa, aplica para 69 entidades disponibles, haciendo alusión en que las mismas, se convierten la segundo tipo de población a estudiar.

\section{Resultados y Análisis}

Con la aplicación de los instrumentos, se identificaron diferentes aspectos, partiendo desde la recopilación de los datos básicos de los graduados, el concepto que se tiene del personal docente y del plan de estudios, seguido de los aspectos en relación al ámbito laboral del graduado, la pertinencia de los contenidos curriculares con el trabajo, entre otros comentarios y sugerencias que apoyen el mejoramiento continuo del programa.

Se identificaron las regiones en las cuales se encuentran actualmente los encuestados, que en su gran mayoría, representados en $97 \%$ continúan en el Departamento Norte de Santander, laborando o estudiando. Se aprecia que el año en que hubo mayor número de graduados, corresponde al segundo semestre de 2015, con un porcentaje de $22 \%$, indicando que en este lapso de tiempo, hubo mayor egreso de estudiantes. Se destaca que un $90 \%$ de los graduados encuestados no interrumpieron sus estudios durante el trascurso de la carrera, lo cual es un indicador positivo para la culminación del proyecto académico.

Dentro de los aspectos que corresponden al plan de 
estudio, se revela como las más valoradas las referidas al contenido curricular y la intensidad horaria, con porcentajes de $76 \%$ y $73 \%$ respectivamente. Los temas que conciernen a las oportunidades de participar en proyectos de investigación, en el énfasis de la investigación durante la enseñanza, y la aplicación de conocimientos al entorno real que percibieron como aspectos por fortalecer.

Se revela que, un $38 \%$ de los graduados se encontraban cursando estudios de formación profesional al momento de desarrollar la encuesta, lo cual les permite complementar sus competencias laborales. Además, se destacó que 24 graduados de los 90 encuestados, se dedican exclusivamente a ejercer su profesión en el ámbito laboral, lo cual es reflejo que la formación recibida contribuyó a que pudieran iniciar en el mercado laboral. Las personas que trabajan y estudian, representados en un $23 \%$, indican resultados favorables para el programa, ya que evidencian una oportunidad de laborar y continuar con su preparación académica.

El hecho de que los encuestados que actualmente estudian se encuentren en la modalidad de pregrado, se debe a que han culminado el programa de tecnología en procesos industriales y han optado por homologar las materias con equivalencia para ingresar a programas como Ingeniería Industrial, o Ingenierías afines. También se encontró que un $6 \%$ de los graduados, han iniciado otros estudios como maestrías y especializaciones, donde se encontrarían ubicados aquellos que pertenecen a las primeras cohortes de graduados del programa.

Las preguntas aplicadas sobre la empleabilidad del graduado permitieron visualizar, que, la mayoría de ellos trabajan, y tardaron menos de seis meses en obtener su primer empleo, valiéndose principalmente de se red de contactos personales y académicas, tal como de muestra en la figura 1.

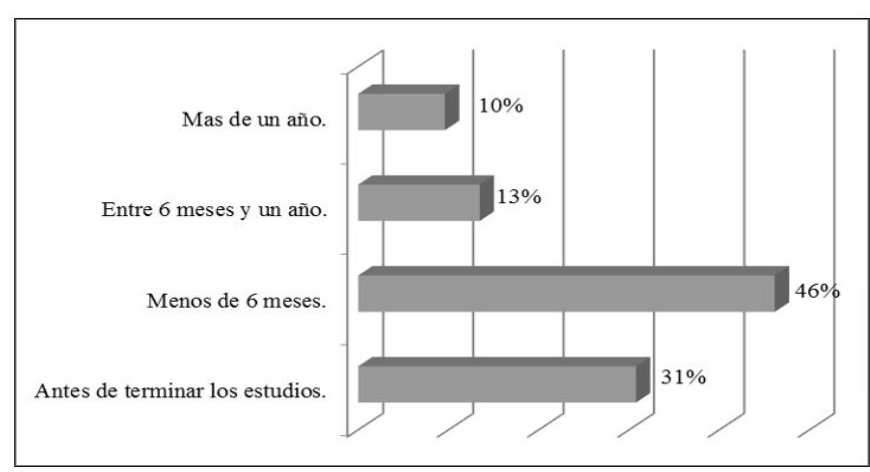

Figura 1. Tiempo invertido para la inserción laboral

Fuente: Autores

Dentro de las pruebas a las que fueron expuestos para la contratación se encuentran pruebas aplicadas sobre conocimiento específico, apoyadas de pruebas psicotécnicas, que apuntan a identificar la personalidad, el entorno psicológico y el rol humana de la persona. Dentro de los requisitos que exigen las empresas para efectos de contratación de tecnólogos, la figura 2 ilustra que los empleadores manifiestan que sus aspirantes deben contar con su título profesional, en un porcentaje de $42 \%$, seguido del énfasis en el uso de paquete de office, software de algún tema en especial; junto con la experiencia profesional. El manejo del segundo idioma, aunque lo destacan como un elemento diferencial de suma importancia, no es pre requisito para la contratación laboral.

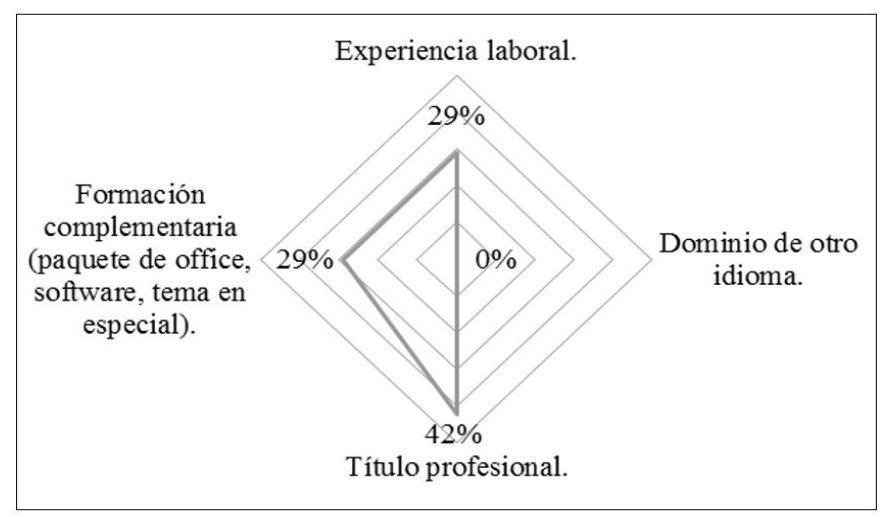

Figura 2. Requisitos para la contratación Fuente: Autores

Sobre las actuales oportunidades de empleabilidad; se vislumbra que el panorama no es del todo favorable, debido a la existencia de fenómenos socioeconómicos en la región que han incidido de forma directa en la dinámica industrial y productiva, lo cual se corrobora por el índice de desempleo en la ciudad, que según el Departamento 
Administrativo Nacional de Estadística DANE [17] ha aumentado hasta en un $18,7 \%$ posicionándose como una de las más altas de país, esto indicaría que a razón de la misma, el graduado percibe que las oportunidades son escazas, lo cual podría mitigarse con fortalecimiento de otro tipo de competencias y habilidades como la innovación y la proyección hacia el emprendimiento. A pesar de lo anterior la población graduada que se encuentra laborando reconoce como una fortaleza la estabilidad laboral, lo que indica que al haber obtenido el empleo, pueden mantenerse en él con facilidad; indicador significativo la Institución porque se correlaciona con la calidad de los procesos académicos desarrollados.

Al indagar sobre el pago seguridad social en las empresas, se encuentra en un $75 \%$ de la muestra, afirma que ha sido cubierta por el empleador. Entre los tipos de contratos más representativos a la hora de emplearse, con un porcentaje de $58 \%$ se refieren a contratos a término fijo menores a un año, y manifiestan tener tiempos inferiores a un año en el empleo actual, donde el salario oscila entre 1-2 salarios mínimos mensuales legales vigentes, lo que deja claro el salario al que se puede aspirar para iniciar labores con esta formación, según su apreciación, lo cual se encuentra dentro de la escala salarial que maneja el país actualmente, pero se puntualiza [18], que debe ser de $\$ 1.185 .000$ para aquellos con contratación a término fijo, con experiencia menor a un año y $\$ 1.754 .500$ para contratación por prestación de servicios, con la misma experiencia que el anterior, lo que superaría los dos salarios mínimos, y señalando que dependiendo de los años de experiencia y manejo de un segundo idioma, los sueldos pueden aumentar.

Posteriormente pudo demostrarse, que los graduados ejercen en puestos como supervisores, cargos pertinentes con la formación recibida y que hacen parte del perfil ocupacional (ver figura 3). Se expone que las empresas donde trabajan los graduados, pertenecen al sector secundario y terciario, categorías de pequeñas y en medianas empresas, lo que es congruente, porque estas en su mayoría están presentes en la región y es significativo que los tecnólogos trabajen en empresas donde pueden contribuir y crecer junto con ellas.

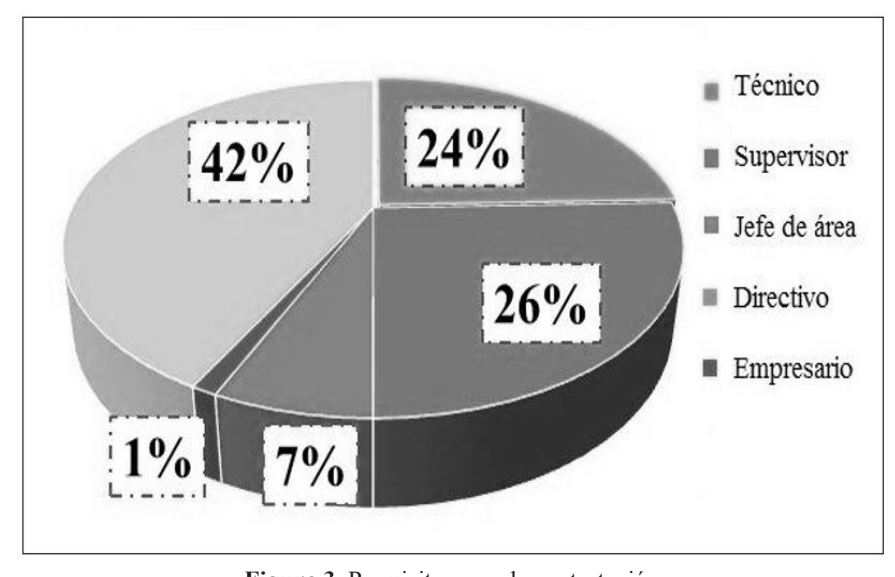

Figura 3. Requisitos para la contratación Fuente: Autores

En relación con el desempeño que han tenido en el ejercicio de la profesión, basado en la coherencia entre la formación y el tipo de empleo, los encuestados coinciden en dar una calificación de buena, destacando la utilidad de los proyectos grado, indicando que están interesados en actualizarse y continuar formándose y reflejando la importancia del manejo de un segundo idioma. Los graduados reconocen que las materias más útiles a la hora de ejercer, son en primer lugar Salud Ocupacional, hoy conocida como Seguridad y Salud en el Trabajo, viendo esta como una necesidad en el mercado, siendo esta la que generó mayor interés y refuerzo en las actividades laborales, seguida de Gestión de la Calidad, Métodos y Tiempos, y Planeación y control de la producción [19] . A razón de lo anterior, se expresa el interés de abordar nuevas asignaturas relacionados con áreas de logística y también el manejo del segundo idioma, específicamente el idioma inglés.

La evaluación del nivel de desempeño de las personas objeto de la investigación, a través de la apreciación del sector productivo de la región, permitió conocer que la muestra utilizada pertenecen en gran mayoría al sector industrial, las cuales ofrecen una contratación a término fijo y con salarios variantes entre uno y dos salarios mínimos, mensuales legales vigentes (SMMLV). Donde el empresario considera que el Tecnólogo en Procesos Industriales cuenta con un perfil adecuado, y que contribuye con la productividad de sus empresas. No obstante, se determinó la posibilidad de implementar acciones de mejora, que permita logar que las asignaturas o áreas del saber posean un enfoque más prácticoaplicado; para ello, se cuenta con disponibilidad de recibir estudiantes semestralmente, permitiendo que estos 
fortalezcan sus conocimientos en el campo productivo, brindando herramientas para que se desenvuelvan con mayor naturalidad y precisión.

Los empleadores del sector productivo enfatizaron que la Universidad y el programa deben procurar buscar mecanismos que permitan mejorar el acompañamiento al sector, y a los estudiantes que realizan proyectos de aula en sus organizaciones para que estos sean eficaces y asumidos con mayor compromiso, lo que podría llevarse a cabo, empleando visitas por parte de los directores de los proyectos, y estableciendo dentro de la agenda institucional, por lo menos una vez al año, una reunión con el empresario, donde se puedan exponer las situaciones de mayor interés para que se establezcan estrategias ajustadas, como las que se presentan en la figura 3.

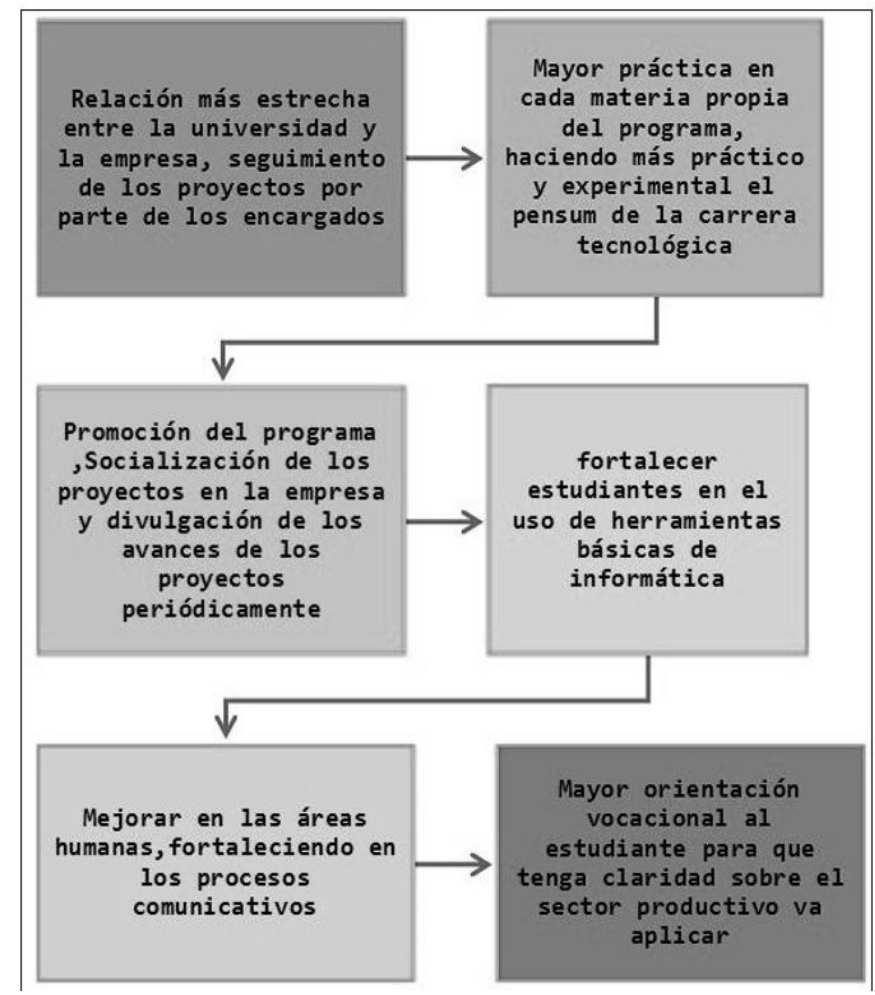

Figura 4. Resultados de encuesta a empleador Aporte del empresario Fuente: Autores

\section{Conclusiones}

A partirdel análisis delos resultados selogró evidenciarque la calidad académica, desde la perspectiva del graduado sobre el programa académico, es significativamente favorable, encontrando notables referencias respecto a la educación que fue impartida a los graduados, en el transcurso de su carrera universitaria, y a su vez reflejando que se requieren mejoras para que exista una mejor capacidad de dar respuesta a las exigencias del mercado laboral. Se determinó que el programa ha realizado acciones muy importantes en la formación de los estudiantes y muestra de ellos es el desempeño de sus graduados y la forma en la cual los empresarios lo reconocen.

La mayor parte de los graduados al terminar sus estudios profesionales tecnológicos continuaron de forma inmediata con el ciclo profesional hacia la Ingeniería. Situación generada, entre otras cosas por la predilección de no aplazar ni detener la continuidad de sus estudios. Igualmente se identificó que los horarios laborales y los horarios de las asignaturas que se ofertan en modalidad diurna se cruzan; lo cual también ha contribuido a que los graduados opten por dar prioridad al proyecto académico.

Es importante señalar según los datos arrojados, pertinentes al desempeño laboral, donde los graduados afirman que las oportunidades de empleo han sido bajas, pero aun así aquellos que obtuvieron un empleo cuentan con las competencias fundamentales intelectuales, interpersonales, personales, organizacionales y tecnológicas para enfrentar cambios y mantenerse en el mercado laboral.

Al conocer las respuestas de los empleadores, se demuestra que los aspectos positivos hacia los estudiantes del programa de Tecnología en Procesos Industriales son muy marcados, oscilando la mayoría entre el $80 \%$ y $90 \%$ de aprobación, reiterando la buena imagen que han dejado los tecnólogos en el sector laboral de la región.

Acorde al estudio realizado, se logró determinar, con las empresas que participaron en el mismo, el conocimiento que tienen sobre el programa de Tecnología en Procesos Industriales, y a su vez el buen concepto del desempeño laboral de sus graduados, hecho destacable, porque genera o aumenta la necesidad de demandar el perfil del Tecnólogo tanto en la parte de proyectos o prácticas, como lo laboral.

Finalmente se hace vital destacar, la importancia de realizar este tipo de estudios, donde se conoce de fuentes que directa o indirectamente se involucran con el proceso de formación, en este escenario el graduado 
y el empleador, porque permiten la toma de decisiones oportunas para la mejora de los procesos de enseñanza, donde las competencias y conocimientos adquiridos, estén cada día más ajustados al contexto real.

\section{Referencias}

[1] M. V. Trigueros, «Importacia de la educación en la actualidad,» Eduinova formación, p. 75, 2010.

[2] OIT Organización Mundial del Trabajo, «Guía para la evaluación de impacto de la formación,» Copyright organización mundial de trabajo (OIT centerfor), vol. 1, n 1, pp. 13-14, 2011.

[3] A. Casanova, «El diseño curricular como factor de calidad,» REICE Revista Iberoamericana sobre Calidad, Eficacia y Cambio en Educación, vol. 10, no 4, p. 334, 19 Septiembre 2012.

[4] C. P. K. B. L. S. C. Nohora Mercado, «Estudio de impacto de los graduados del programa de ingenieria de la costa,» Barranquilla, 2013.

[5] MEN Ministerio Nacional de Educación, «Definición de Competencias Laborales».

[6] M. C. Rita Bustos, «La evaluación de impacto: una alternativa de investigación.,» Revista Unimonserrate, $\mathrm{n}^{\circ}$ 9, pp. 3-6, 15 Junio 2012.

[7] G. J. David, "Análisis de la pertinencia del Programa cademico de Ingeniería Civil,» pp. 6-8, 2011.

[8] Y. A. Alex Lozano, «Perfil Ocupacional y profesional de los graduados de la especialización en Administración Financiera de la EAN,»pp. $1-8,2014$.

[9] D. Rojas, «Impacto en la situación laboral de graduados de la educación presencial y virtual estudio comparativo,» Universidad de la Sabana, vol. 17, $\mathrm{n}^{\mathrm{o}}$ 2, p. 3, 8 Julio 2014.
[10] Consejo Nacional de Acreditación CNA, «Lineamientos para la acreditación de programas de Pregrado,» pp. 46-47, 2013.

[11] F. M. V. M. C. G. B. R. M. Ramos Azcuy, «Instrumento para la evaluación del impacto de la formación académica,» Revista Universidad y Sociedad [seriada en línea], vol. 8, n 2 2, pp. pp. 114-124, 2016.

[12] Incotec Internacional, «Sistema de Gestión de Calidad,» vol. 2, pp. 2-3, 2015.

[13] FUOC. Fundación para la Universitat Oberta de Catalunya, «Herramientas de medición de impacto social,» vol. 3, s.f.

[14] Consejo Nacional de Acreditación CNA, «Lineaminetos para la Acreditación de Programas de Pregrado,» Enero 2013.

[15] S. A. G. Eucario Parra Castrillón, «Metodología para caracterización y estudio de impacto en el medio de graduados de instituciones de educación,» Revista Interamericana de Educación, Pedagogía, y estudios culturales, pp. 84-86, 2016.

[16] C. F. M. d. P. B. Roberto Hernández, Metodología de la investigación, vol. 5, J. M. Chacón, Ed., Mexico : McGRAW-HILL / Interamericana editores, , S.A. DE C.V., 2010.

[17] El espectador, «El desempleo subio en febrero de 2018 en un 10,8\%,» El desempleo, 28 Marzo 2018.

[18] Enlace Profesional, «Escala de Minimos de Remuneración 2018,» 2018.

[19] P. G. P. G. Yoneidy Benítez, Resultados de encuesta sobre empleabilidad, Cucuta, 2018.

[20] Deming. [En línea]. Available: http:// mejoracontinua.net/que-es-la-mejora-continua- 
Impacto laboral del graduado del programa de tecnología en procesos industriales de la Universidad Francisco de Paula Santander segun-autores. [Último acceso: 16 Marzo 2018]. 